\title{
Development of a Master Sintering Curve for Al-Mg Alloy
}

\author{
Yong-Shin Lee, ${ }^{1}$ Sang-Jun Lee, ${ }^{2}$ Sangmok Lee, ${ }^{3}$ Eung-Zu Kim, ${ }^{3}$ and Duck Jae Yoon ${ }^{3}$ \\ ${ }^{1}$ School of Mechanical Engineering, Kookmin University, 77 Jeongneung-ro, Seongbuk-gu, Seoul 02707, Republic of Korea \\ ${ }^{2}$ Patent Information Promotion Center, 48 Munjeong-ro, 48-Beon-gil, Seo-gu, Daejeon 35262, Republic of Korea \\ ${ }^{3}$ Metal Forming R\&D Group, Korea Institute of Industrial Technology, Incheon 21999, Republic of Korea
}

Correspondence should be addressed to Yong-Shin Lee; yslee@kookmin.ac.kr

Received 27 June 2016; Accepted 25 August 2016

Academic Editor: Sutasn Thipprakmas

Copyright (C) 2016 Yong-Shin Lee et al. This is an open access article distributed under the Creative Commons Attribution License, which permits unrestricted use, distribution, and reproduction in any medium, provided the original work is properly cited.

\begin{abstract}
A new master sintering curve (MSC) is proposed for Al-Mg alloy in order to effectively design the pressure-assisted sintering process. In this work, hot pressing experiments of Al-Mg alloy powders are performed. The changes of relative density during hot pressing are measured for the various heating rates of $5^{\circ} \mathrm{C} / \mathrm{min}, 10^{\circ} \mathrm{C} / \mathrm{min}$, and $20^{\circ} \mathrm{C} / \mathrm{min}$ at the fixed pressure of $50 \mathrm{MPa}$. A work of sintering, designated as $\Theta$, is introduced and defined as $\Theta(t, T)=\int_{0}^{t}(1 / T) \exp (-Q / R T) d t$. A work of sintering, $\Theta$, could be interpreted as a measure for the amount of sintering work. The MSC in this work defines the relation between the apparent density and a work of sintering, $\Theta$. Since the measurement of an apparent activation energy, $Q$, is very difficult, the correct value of $Q$ is obtained numerically using a mean residual square method. Then, the master sintering curves for sintering of Al-Mg alloy powders are proposed for the sintering temperatures of $400^{\circ} \mathrm{C}$ and $500^{\circ} \mathrm{C}$ through scaling procedures. It is expected that the master sintering curves proposed in this work could help an engineer to design pressure-assisted sintering process for $\mathrm{Al}-\mathrm{Mg}$ alloy.
\end{abstract}

\section{Introduction}

The demand for more light weight, fuel efficient, and enhanced performance automobiles has stimulated research and development in high-strength and high formability aluminum alloys. The Al-Mg alloys have medium to high strength, excellent formability, and good corrosion resistance [1]. However, it has been well known that sintering with the powder of $\mathrm{Al}-\mathrm{Mg}$ alloys is not easy.

In the conventional design procedure of sintering, the parameters such as time and temperature of the sintering are decided mainly on the "trial and error" basis [2]. If the densification during sintering could be predicted from the sintering data that are really available, it would be helpful for engineers to design a new sintering process. Then, the sintering strategy could be established on that basis, which leads to the production of pellets of good quality and less number of defects. Therefore, there is a need for better understanding of a whole sintering procedure. The theory of master sintering curve (MSC) provides a new insight into the understanding of sintering process [3-5].

The master sintering curve enables engineers to predict the densification behavior under arbitrary time-temperature schedules with the help of a minimum of preliminary experiments. However, this curve has been known as sensitive to the factors such as starting morphology of the powder, fabrication route, dominant diffusion mechanism, and heating condition used for sintering [6]. Thus, one may need a new MSC according to the changes of such factors if any of them is changed when designing a sintering process. A MSC can also be used as an aid to compare the sinterability of different powders and to examine the effects of additives, atmosphere, and fabrication procedure of sintering. Recent study could incorporate the effects of pressure on densification during sintering into one MSC such that the final density at any applied pressure in a pressure-assisted sintering can be predicted [2]. The concept of MSC, which was originally developed for the sintering of ceramic powders, has been successfully extended to the sintering with metallic powders such as $\mathrm{Al}_{2} \mathrm{O}_{3}, \mathrm{XnO}$, rhenium, and nickel powders [2-7]. More recently, pressure-assisted sintering of hard metallic powders such as tungsten alloys and WC alloys has been reported [8, 9]. For instance, An [9] constructed the master sintering curves for the mixture of $\mathrm{WC}, \mathrm{Cu}$, and Ni powders to understand densification behaviors during the resistance 
heating. However, the extension of the concept of MSC to sintering with various metallic powders is still in early stage. The motivation for the present study is to extend the concept of the master sintering curve into the realm of hot pressing with metallic powders and to establish the pressure-assisted master sintering curve of $\mathrm{Al}-\mathrm{Mg}$ alloy.

\section{Theory of a Master Sintering Curve}

Sintering of particulate materials is a thermally activated process involving the mass-transport phenomena that would result in particle bonding [10]. These mass-transport phenomena are generally to reduce the free surface area in the compact of particles. On the other hand, the surface transport mechanisms are to only grow the sinter bonding between particles. Thus, it is the bulk transport mechanisms that increase the density of porous body by gathering the particle together in order to reduce the free surface energy. Associated with this sintering densification is a decrease in volume, so the changes of density can be monitored by measuring dimensional changes of a compact during sintering. Hansen et al. [11] developed a combined-stage sintering model with the extensive studies on the mass-transport mechanisms during sintering, which is able to predict both the dimensional and density changes that take place during sintering. In their work, the compact densification is related to the dominant diffusional events such as grain boundary and volume diffusion, resulting in the following combined-stage sintering equation:

$$
-\frac{d L}{L d t}=\frac{\gamma \Omega}{k T}\left[\frac{\Gamma_{v} D_{v}}{G^{3}}+\frac{\Gamma_{b} D_{b}}{G^{4}}\right],
$$

where $L$ is a representative dimension of the compact, $t$ is time, $\gamma$ is the surface energy, $\Omega$ is the atomic volume, $k$ is Boltzmann's constant, $T$ is the temperature, $\Gamma$ is a lumped scaling factor, $D$ is the diffusivity, $G$ is the grain size, and the subscripts $v$ and $b$ refer to volume diffusion and grain boundary diffusion, respectively.

For an isotropic shrinkage, the sintering shrinkage rate can be linked to the densification rate by considering the conservation of mass. Then, the following approximate relationship could be obtained:

$$
-\frac{d L}{L d t}=\frac{d \rho}{3 \rho d t} .
$$

Combining (1) and (2) provides a link between the densification rate and the diffusional sintering phenomena. However, the lumped scaling factors, $\Gamma_{v}$ and $\Gamma_{b}$, are arbitrary parameters containing the density-dependent geometric terms. Furthermore, the diffusivities for grain boundary and volume diffusion, $D$, vary significantly between the studies [12]. These are the main reasons why no reliable value can be assigned confidently to a particular material. Thus, determination of the shrinkage and densification rates from the combined-stage sinter model requires experimental characterization of the lumped scaling factors and diffusivities for specific sintering systems. In order to overcome this difficult work, Su and Johnson [3,6] tried to rearrange the combined-stage sintering equation by grouping all of the density-dependent material parameters on one side, while the known process-dependent parameters, with the exception of the apparent activation energy $Q$, grouped on the other side:

$$
\frac{k}{\gamma \Omega_{a} D_{0}} \int_{\rho_{0}}^{\rho} \frac{(G(\rho))^{n}}{3 \rho \Gamma(\rho)} d \rho=\int_{0}^{t} \frac{1}{T} \exp \left(-\frac{Q}{R T}\right) d t .
$$

Here, $D_{0}$ is the diffusivity preexponent. $D_{0}$ and $n$ depend on the dominant sintering mechanism. The diffusivities as given in (1) follow Arrhenius temperature dependence and have been broken up into their preexponential and exponential terms in (3). Since the grain boundary and volume diffusion have diverse influences on the densification response of a sintering system, they have been lumped together as an apparent diffusivity in (3) to create a master sintering curve. Here, $D_{0}$ and $n$ are determined as $D_{0}=\left(D_{b}\right)_{0}$ and $n=4$ for grain boundary diffusion and $D_{0}=\left(D_{v}\right)_{0}$ and $n=3$ for volume diffusion. The rearranged combinedstage sintering model would be integrated from the initial time to the current time for the right-hand side. Similarly, the left-hand side could be evaluated by integrating the function represented in terms of a density from the initial density to the current density which can be obtained at any state of $(t, T, \rho)$ during thermal processing. The right-hand side of (3) could be interpreted as a measure of the energy supplied to the system during nonisothermal sintering. Correspondingly, the left-hand side might be interpreted as the increase of internal energy due to the changes of density during sintering.

Now, the term $(\Theta)$, called a work of sintering in this work, is introduced as follows:

$$
\Theta(t, T)=\int_{0}^{t} \frac{1}{T} \exp \left(-\frac{Q}{R T}\right) d t .
$$

The apparent activation energy for sintering in (4) could be found through a numerical analysis, such as mean square residual method, the detailed procedure of which will be shown in the following section. Now it is clear that there must be an apparent density corresponding to a work of sintering, $\Theta$, at a given time $t$. Once the relationship between the work of sintering and the relative density is established, a master sintering curve describing this relationship could be constructed using a scaling procedure or curve fitting.

\section{Numerical Procedure to Construct a MSC}

As mentioned previously, the MSC model is derived from the final equation of the combined-stage sintering model which is known as an even earlier attempt at the quantitative prediction of sintering behavior known. However, the MSC model offers a simpler method to predict the final density of a sintered product. The value of apparent activation energy is necessary to evaluate a work of sintering $(\Theta)$ in (4). But the accurate value of apparent activation energy (Q) is not known for many cases. Su and Johnson introduced the fitting procedure to obtain the proper value of apparent activation energy $(Q)$ using the relationships between a work of sintering and density for at least three different heat 
rates in sintering [6]. This procedure starts with an initially assumed value of $Q$. Then, the values of $\Theta$ and the density at the time $t$ during sintering are evaluated with the assumed value of $Q$ and they are plotted on the domain of $\rho$-Q. When the assumed values of $Q$ are appropriate, then all the points corresponding to $(\rho, Q)$ could be merged onto one curve. In this study, the proper value of apparent sintering activation energy is found out by minimizing the normalized dimensionless mean residual square, defined as the following equation as suggested by $\mathrm{Su}$ and Johnson [6]:

Mean residual square

$$
=\sqrt{\frac{1}{\rho_{f}-\rho_{o}} \int_{\rho_{o}}^{\rho_{f}} \frac{\sum_{i=1}^{N}\left(\Theta_{i} / \Theta_{\mathrm{avg}}-1\right)^{2}}{N} d \rho}
$$

where $N$ is the number of experimental data, $\Theta_{i}$ is the work of sintering at $i$ th experimental measurement, $\Theta_{\text {avg }}$ is the average value of all $\Theta_{i}$ over $N$, and $\rho_{f}$ and $\rho_{o}$ are the final density and initial density, respectively.

When the activation energy obtained here would be compared to those reported in the literatures for the activation energies associated with specific diffusional paths, that is, volume, surface, or grain boundary diffusion, the primary diffusional mechanisms in this sintering would be identified as explained in the others [10, 13]. Now, a MSC can be constructed by plotting the relative density, which would be extracted from experimental dilatometry data, against the natural logarithm of the work of sintering represented by (4). Again, all data points of $(\rho, \Theta)$ obtained with the accurate activation energy are merged onto one master curve or a master sintering curve. The sintering activation energy used in this calculation is temperature-dependent. There have been several tries to represent such complicated shape of a MSC with various functions. Su and Johnson invoked a polynomial function to describe the master sintering curve relationship [6]. However, a polynomial function generally requires many coefficients to precisely represent the complicated shape of a MSC. In order to reduce the material constant needed for a given MSC, it is a better way to invoke the function similar to the complicated curve shape. Teng et al. [14] and Blaine et al. [15] developed the new functions which can represent s sigmoidal shape. Teng et al. used three unknown parameters, but Blaine et al. used only two unknown parameters. In this study, we used Teng's sigmoidal function to better match the complicated master sintering curve of Al-Mg alloy, defined as the following equation:

$$
\rho=\rho_{o}+\frac{a}{\left[1+\exp \left(-\left(\ln \Theta-\ln \Theta_{o}\right) / b\right)^{c}\right]},
$$

where $a, b$, and $c$ are the material constants. It should be mentioned that the material constants $a$ and $c$ coincide with the point of inflection of the curve while the constant $b$ is the slope of the linearized portion. The overall procedure for the construction of master sintering curve is summarized in Figure 1.
TABLE 1: Chemical compositions of the Al-Mg alloy in weight percent.

\begin{tabular}{lcccccccr}
\hline Alloy & $\mathrm{Mg}$ & $\mathrm{Mn}$ & $\mathrm{Zn}$ & $\mathrm{Si}$ & $\mathrm{Cu}$ & $\mathrm{Ni}$ & $\mathrm{Fe}$ & $\mathrm{Al}$ \\
\hline Al-Mg & 4.00 & 0.59 & 0.03 & 0.15 & 0.39 & 0.02 & 0.24 & $\mathrm{Bal}$. \\
\hline
\end{tabular}

TABLE 2: Experimental schedules for hot pressing of Al-Mg alloy powders.

\begin{tabular}{|c|c|c|c|}
\hline Sintering temperature $\left[{ }^{\circ} \mathrm{C}\right]$ & & 400 & \\
\hline Holding time [min] & & 30 & \\
\hline Sintering pressure $[\mathrm{MPa}]$ & & 50 & \\
\hline Heating rate $\left[{ }^{\circ} \mathrm{C} / \mathrm{min}\right]$ & 5 & 10 & 20 \\
\hline Lubricant & & Graphite foil & \\
\hline
\end{tabular}

(a) 1st schedule.

\begin{tabular}{|c|c|c|c|}
\hline Sintering temperature $\left[{ }^{\circ} \mathrm{C}\right]$ & & 500 & \\
\hline Holding time [min] & & 0 & \\
\hline Sintering pressure $[\mathrm{MPa}]$ & & 50 & \\
\hline Heating rate $\left[{ }^{\circ} \mathrm{C} / \mathrm{min}\right]$ & 5 & 10 & 20 \\
\hline Lubricant & & ite foil & \\
\hline
\end{tabular}

(b) 2nd schedule.

\section{Hot Pressing Experiments of Al-Mg Alloy}

In order to develop a MSC of Al-Mg alloy, hot pressing experiments are carried out. The chemical compositions of the Al-Mg alloy used in experiments are given in Table 1. Al$\mathrm{Mg}$ alloy powder having an average particle size of $45 \mu \mathrm{m}$ filled a graphite mold in glove box for a hot pressing. The pellet was preformed at room temperature and under the uniaxial compression of $10 \mathrm{MPa}$. Its diameter and height are $20 \mathrm{~mm}$ and $13.5 \mathrm{~mm}$, respectively. Each sample was pressed using Al-Mg alloy powders which weighted about 8 grams. The average density of a bulk body was calculated as $\rho=$ $m / V$, where $m$ is the mass and $V$ is the volume. It must be mentioned that the densities in a bulk body are assumed as distributed uniformly. The experimental schedules for hot pressing experiments are summarized in Table 2. In order to properly construct a MSC, various experimental conditions were considered. The most important parameters in hot pressing are the peak temperature and its holding time. In this work, two schedules are considered: (1) peak temperature $400^{\circ} \mathrm{C}$ and holding time $30 \mathrm{~min}$ and (2) peak temperature $500^{\circ} \mathrm{C}$ and no holding time. Experiments with the above two schedules are performed under the various heating rates and pressures. The constant heating rates considered in this work are $5^{\circ} \mathrm{C} / \mathrm{min}, 10^{\circ} \mathrm{C} / \mathrm{min}$, and $20^{\circ} \mathrm{C} / \mathrm{min}$. The sintering pressure of $50 \mathrm{MPa}$ is examined. In experiments, the cooling was done in the furnace until the room temperature. The experimental apparatus of hot pressing in this work is newly developed and is schematically shown in Figure 2.

\section{Construction of Master Sintering Curves for Al-Mg Alloy}

First, the relative density could be evaluated based on the punch advancement in experiments and would be plotted 


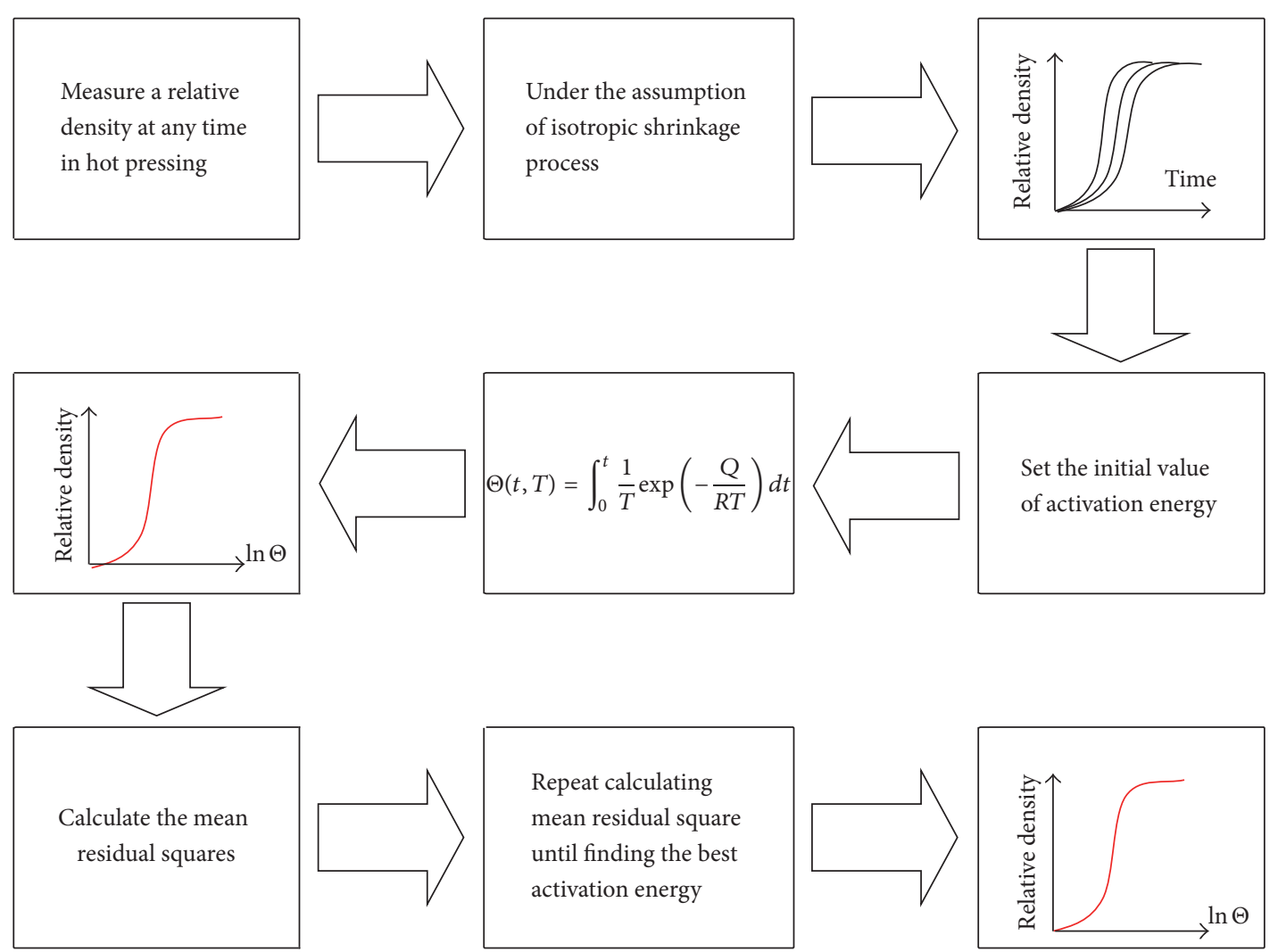

FIGURE 1: The overall procedure for the construction of a master sintering curve.

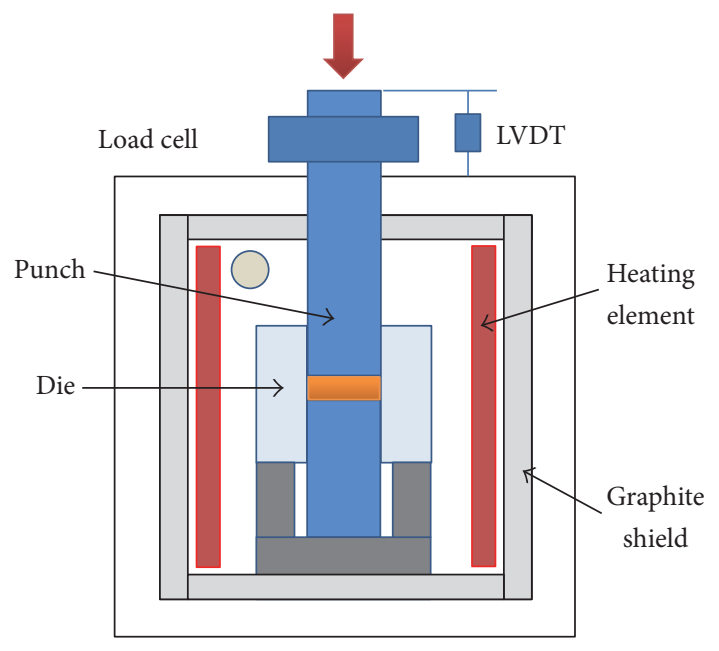

Figure 2: Schematic drawing of the hot press apparatus.

with respect to time during sintering. The densification curves, which represents the relative density changes during sintering, allow engineers to understand the characteristics of densification during sintering. Figure 3 shows various densification curves for various hot pressing conditions. Those are Case (a) sintering temperature of $400^{\circ} \mathrm{C}$, pressure of $50 \mathrm{MPa}$, and holding time of $30 \mathrm{~min}$ and Case (b) sintering temperature of $500^{\circ} \mathrm{C}$, pressure of $50 \mathrm{MPa}$, and no holding time. Three different heating rates of 5,10 , and $20^{\circ} \mathrm{C} / \mathrm{min}$ are examined. As shown in Figure 3, a densification curve may be divided into three sections. In the first section, the initial relative density holds until densification is activated. In the second section, the densification starts with the high rate of densification and this rate holds until the relative reaches about $95 \%$. In the third section, the rate of densification becomes slow. Two intersection points are observed between 1st and 2nd sections and between 2nd and 3rd sections. It is observed that the first intersection point, the temperature for the initiation of visible densification, varies for three heating rates. Although the effect of pressure on the sintering process is not given specifically, sintering pressure of $20 \mathrm{MPa}$ was examined in experiments. As expected, the pressure is shown to facilitate sintering by showing the fast appearance of the above intersection points. In experiments, temperature holding time is used as a parameter since it affects the sintering time. However, it should be noted that the important factor is not a temperature holding time but an exposure time to thermal activation or sintering time, which can induce the densification during sintering process. In high heating rate of $20^{\circ} \mathrm{C} / \mathrm{min}$, the exposure time without temperature holding time is much shorter than that with temperature holding time. Thereby, the final densification without temperature holding time in Figure 3(b) does not reach that with temperature holding time in Figure 3(a). In slow heating rate of $5^{\circ} \mathrm{C} / \mathrm{min}$, the sintering time with high peak temperature of $500^{\circ} \mathrm{C}$ is slightly shorter than that with temperature holding time at the low peak temperature of $400^{\circ} \mathrm{C}$. As shown in Figure 3 for heating rate of $5^{\circ} \mathrm{C} / \mathrm{min}$, their densification 


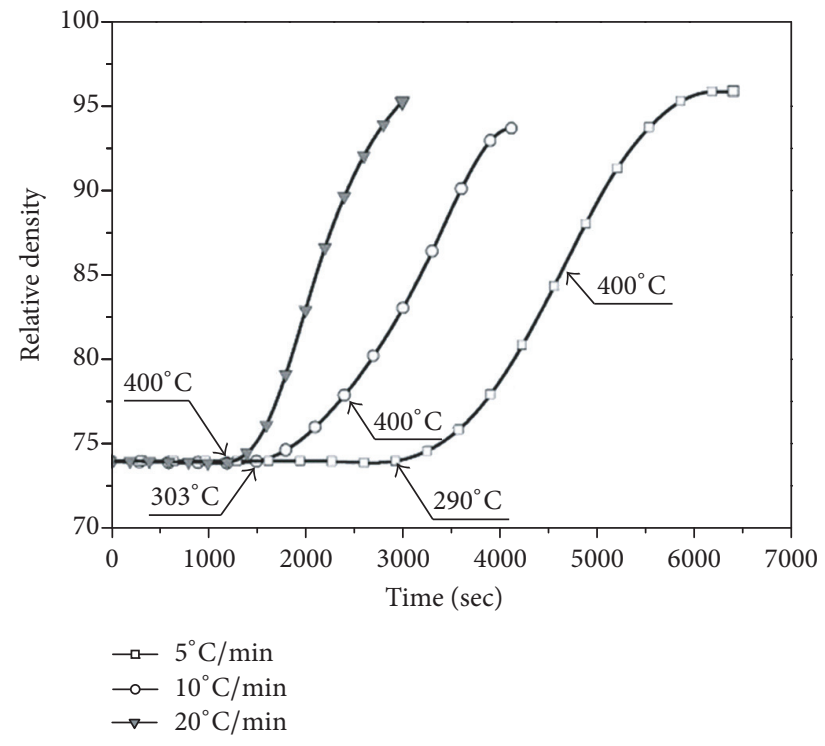

(a)

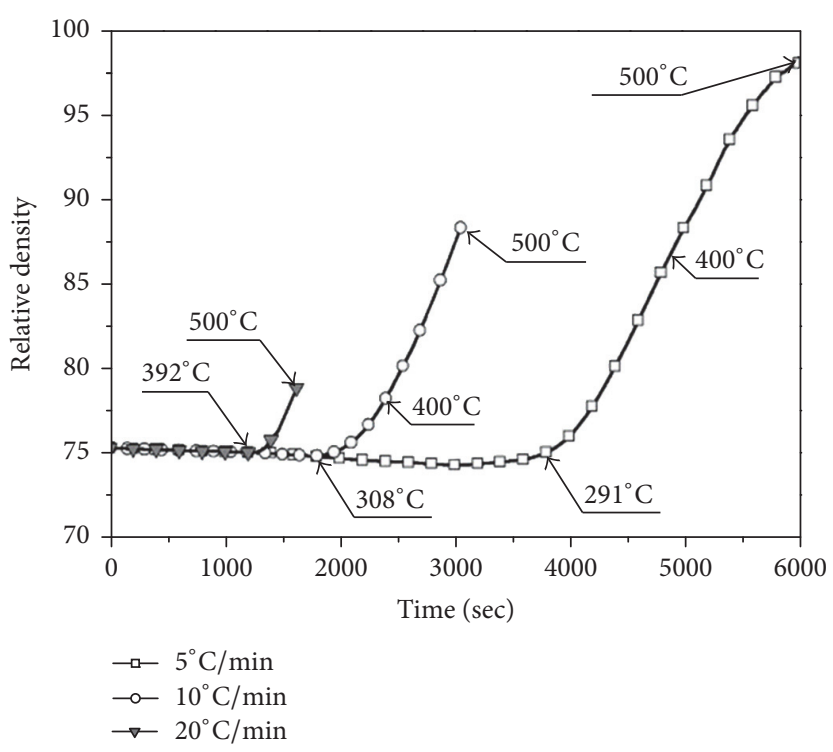

(b)

FiguRE 3: Densification curves: (a) sintering temperature of $400^{\circ} \mathrm{C}$, pressure of $50 \mathrm{MPa}$, and holding time of 30 min; (b) sintering temperature of $500^{\circ} \mathrm{C}$, pressure of $50 \mathrm{MPa}$, and no holding time.

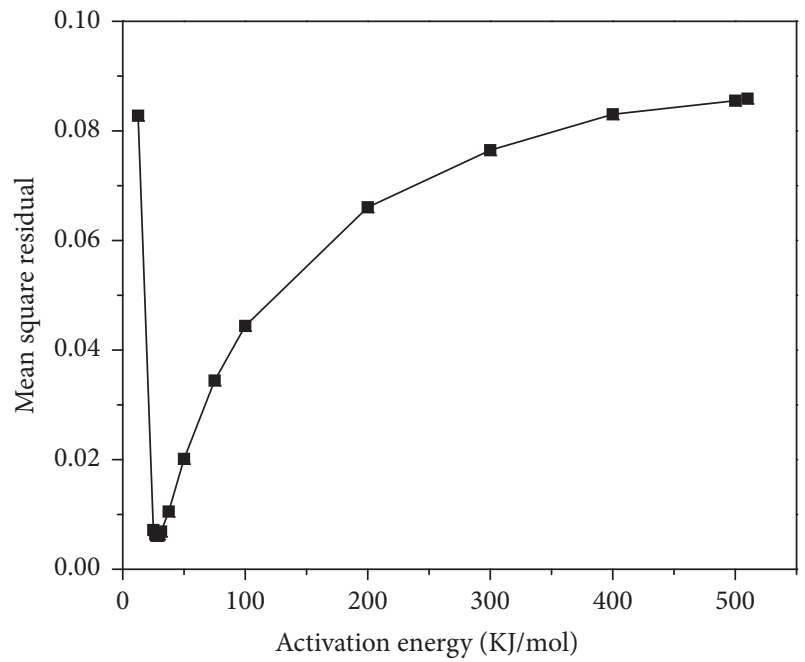

(a)

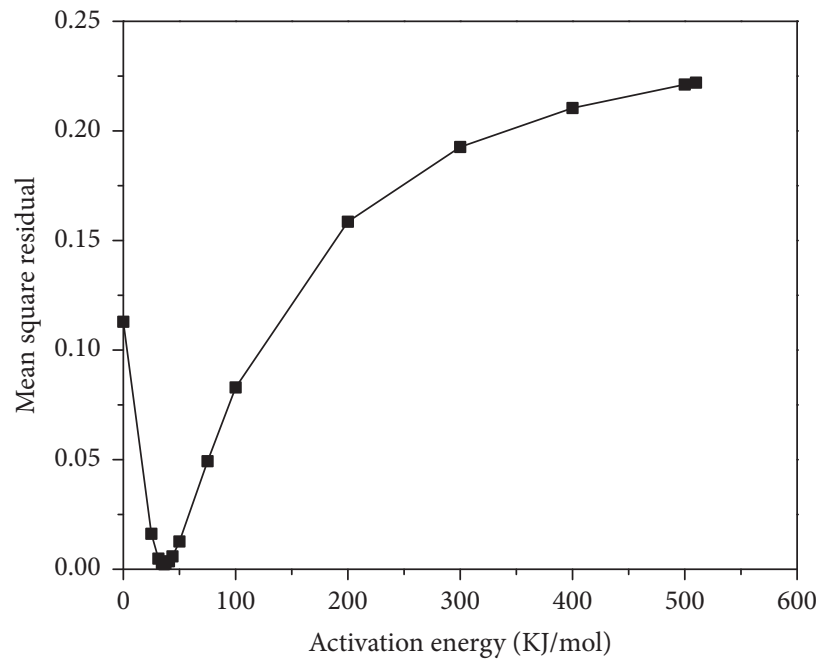

(b)

FIGURE 4: Mean square residual versus apparent activation energy: (a) sintering temperature of $400^{\circ} \mathrm{C}$, pressure of $50 \mathrm{MPa}$, and holding time of $30 \mathrm{~min}$; (b) sintering temperature of $500^{\circ} \mathrm{C}$, pressure of $50 \mathrm{MPa}$, and no holding time.

behaviors are similar because the difference in the sintering time or exposure time to thermal activation between them is not so long. But the final density with the peak temperature of $500^{\circ} \mathrm{C}$ is slightly higher than that with the peak temperature $400^{\circ} \mathrm{C}$ although the exposure time with the peak temperature of $500^{\circ} \mathrm{C}$ is shorter. Thereby, one may say that the higher peak temperature could result in higher densification rate. Now, it may be concluded that the important factors in pressureassisted sintering of $\mathrm{Al}-\mathrm{Mg}$ alloy are the exposure time to thermal activation and the peak temperature.

In order to find a correct activation energy $Q$, an initial estimate for $Q$ must be made. In this work, $500 \mathrm{KJ} / \mathrm{mol}$ is used as initial guess, which is much bigger than the correct values given in the literatures. Then, the work of sintering $(\Theta)$ at the time, when the relative density was measured in experiments, can be computed using (4) for three heating rates of 5,10 , and $20^{\circ} \mathrm{C} / \mathrm{min}$. This procedure is repeated with the new value of activation energy $Q$, smaller than the current one, until $Q$ reaches the value, close to zero such as 1 . In this work, more than 50 values of $Q$ are examined. The mean square residuals corresponding to those values of $Q$ are calculated according to (5). Then, the variations of mean square residual corresponding to $Q$ are plotted as given in Figures 4(a) and 4 (b). It should be mentioned that the correct value of $Q$ is obtained at the minimum of the mean square residual. 


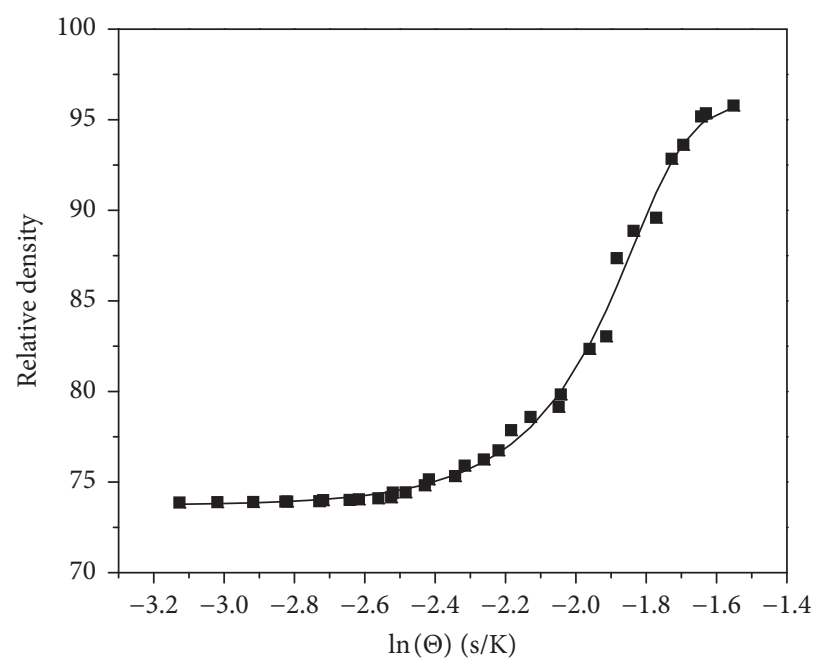

(a)

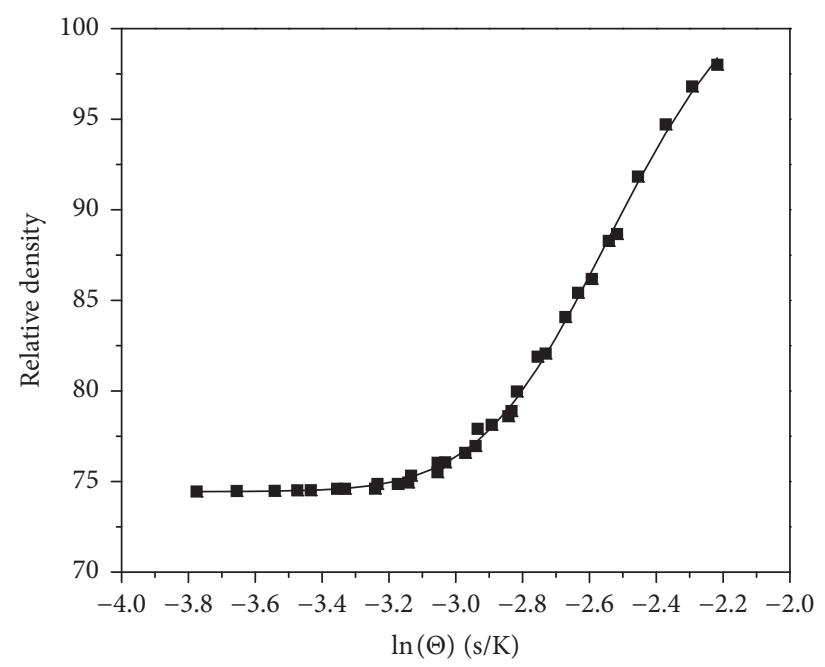

(b)

Figure 5: Master sintering curves: (a) sintering temperature of $400^{\circ} \mathrm{C}$, pressure of $50 \mathrm{MPa}$, and holding time of $30 \mathrm{~min}$; (b) sintering temperature of $500^{\circ} \mathrm{C}$, pressure of $50 \mathrm{MPa}$, and no holding time.

Once the correct value of $Q$ is given, then a master curve could be obtained that represents the relationship between the relative density at any time during sintering and the work of sintering until that time, defined by (4). When the relative density during sintering is plotted against the logarithm of a sintering work or $\ln (\Theta)$, it appears in a form of sigmoidal function given in (6).

As for Case (a) with three heating rates of 5,10 , and $20^{\circ} \mathrm{C} / \mathrm{min}$, the minimum of $Q$ is found out as $28 \mathrm{KJ} / \mathrm{mol}$ as shown in Figure 4(a). Now, the other densification curve shown in Figure 3(a) could be merged into one master curve as shown in Figure 5(a). As shown in Figure 4(b), the minimum of $Q$ is $36.4 \mathrm{KJ} / \mathrm{mol}$ for Case (b). The corresponding master sintering curve is given in Figure 5(b). The maximum errors between the predicted relative density by a master sintering curve and the measured density in experiments are less than $1 \%$ as shown in Figure 5. This implies that the concept of a master sintering curve can be successfully extended into the realm of hot pressing of Al-Mg alloy.

It should be noted that the values of activation energy obtained in this study are slightly bigger than that $(Q=$ $25.0 \mathrm{KJ} / \mathrm{mol}$ ) by Witkin et al. [16], although those values are generally below the activation energies of aluminum lattice with the main grain boundary self-diffusion mechanism reported in the literatures $[17,18]$. In fact, the activation energy is very sensitive to the sintering conditions such as the preparing procedure of green compacts, the sintering procedure, applied pressure, peak temperature, and its holding time, even for the same material.

Now, the coefficients of the master sintering curves as represented in (6) are summarized in Table 3. The individual sintering points converged reasonably close to single curve. This indicates that there should have been a general curve, master sintering curve, which is independent of the sintering path or heating rates. It suggests that integration of a proposed sintering time-temperature profile yields a point on the
TABLE 3: The coefficients in the master sintering curves for $\mathrm{Al}-\mathrm{Mg}$ alloy powders.

\begin{tabular}{lcccc}
\hline \multicolumn{4}{c}{$a$} & \multicolumn{4}{c}{$a$} & \\
& $\rho_{s}=\rho_{0}+\frac{}{\left[1+\exp \left(-\left(\ln \Theta-\ln \Theta_{0}\right) / b\right)^{c}\right]}$ & \\
Condition & $\rho_{0}$ & $a$ & $b$ & $c$ \\
\hline$P=50 \mathrm{MPa}$ \\
$\begin{array}{l}\text { Temperature } 400^{\circ} \mathrm{C} \\
\text { Holding time } 30 \mathrm{~min}\end{array}$ & 73.72 & 22.38 & 0.07 & 0.32 \\
\hline$P=50 \mathrm{MPa}$ & & & & \\
Temperature $500^{\circ} \mathrm{C}$ & 74.43 & 31.09 & 0.26 & 2.26 \\
No holding time & & & & \\
\hline
\end{tabular}

master sintering curve. The expected density can be obtained by finding the ordinate value at that point. On the other hand, if the final desired density is known, it is possible to obtain the corresponding $\Theta$ value from the abscissa of the master sintering curve and therefore to design the sintering schedule.

\section{Conclusions}

This study is concerned with the development of master sintering curves for $\mathrm{Al}-\mathrm{Mg}$ alloy, which can provide a useful method to quantify the densification behavior during sintering. Hot pressing of Al-Mg alloy powders is performed under the constant heating rate at 5,10 , and $20^{\circ} \mathrm{C} / \mathrm{min}$ for two schedules: (1st schedule) peak temperature $400^{\circ} \mathrm{C}$ and holding time $30 \mathrm{~min}$; (2nd schedule) peak temperature $500^{\circ} \mathrm{C}$ and no holding time. The activation energy, $Q$, is obtained as $28.0 \mathrm{KJ} / \mathrm{mol}$ for the 1 st schedule, while $Q=36.4 \mathrm{KJ} / \mathrm{mol}$ for the 2nd schedule. It is shown that the concept of master sintering curve could be successfully extended into the realm of hot pressing of $\mathrm{Al}-\mathrm{Mg}$ alloy by constructing a master sintering curve for $\mathrm{Al}-\mathrm{Mg}$ alloy. The constructed master sintering curves according to $Q=28.0$ and $36.4 \mathrm{KJ} / \mathrm{mol}$ for the sintering 
temperatures of $400^{\circ} \mathrm{C}$ and $500^{\circ} \mathrm{C}$, respectively, show the maximum error of less than $1 \%$. Finally, it is concluded that the master sintering curves presented in this work would be helpful for engineers to design a new sintering process of AlMg alloy.

\section{Competing Interests}

The authors declare that they have no competing interests.

\section{References}

[1] L. Zhen and S. B. Kang, "The effect of pre-aging on microstructure and tensile properties of Al-Mg-Si alloys," Scripta Materialia, vol. 36, no. 10, pp. 1089-1094, 1997.

[2] D. L. Johnson and H. Su, "A practical approach to sintering," American Ceramic Society Bulletin Journal, vol. 76, p. 72, 1997.

[3] D. L. Johnson and H. Su, “The master sintering curve," Advances in Powder Metallurgy and Particulate Materials, vol. 2, p. 115, 1997.

[4] D. L. Johnson, M. Henrichsen, J. Hwang, and V. P. Dravid, "Ultra rapid phase conversion in $\beta^{\prime \prime}$-alumina tubes," Journal of the American Ceramic Society, vol. 83, no. 11, pp. 2861-2862, 2000.

[5] M. Stöcker, "From rapid prototyping to rapid manufacturing," Auto Technology, vol. 2, p. 38, 2002.

[6] H. Su and D. L. Johnson, "Master sintering curve: a practical approach to sintering," Journal of the American Ceramic Society, vol. 79, no. 12, pp. 3211-3217, 1996.

[7] W. Q. Shao, S. O. Chen, D. Li, H. S. Cao, T. C. Zhang, and S. S. Zhang, "Prediction and control of microstructure evolution for sub-microscale $\alpha-\mathrm{Al}_{2} \mathrm{O}_{3}$ during low-heating-rate sintering based on the master sintering curve theory," Journal of the European Ceramic Society, vol. 29, no. 1, pp. 201-204, 2009.

[8] S. J. Park, J. L. Johnson, Y. Wu, Y.-S. Kwon, S. Lee, and R. M. German, "Analysis of the effect of solubility on the densification behavior of tungsten heavy alloys using the master sintering curve approach," International Journal of Refractory Metals and Hard Materials, vol. 37, pp. 52-59, 2013.

[9] K. J. An, "Application of the pressure-assisted master sintering surface," Metals and Materials International, vol. 20, no. 1, pp. 113-118, 2014.

[10] R. M. German, Sintering Theory and Practice, John Wiley \& Sons, New York, NY, USA, 1996.

[11] J. D. Hansen, R. P. Rusin, M. -H. Teng, and D. L. Johnson, "Combined-stage sintering model," Journal of the American Ceramic Society, vol. 75, no. 5, pp. 1129-1135, 1992.

[12] I. Kaur, W. Gust, and L. Kozma, Handbook of Grain and Interphase Boundary Diffusion Data, 1, University of Stuttgart, 1996.

[13] J. Wang and R. Raj, "Estimate of the activation energies for boundary diffusion from rate-controlled sintering of pure alumina, and alumina doped with zirconia or titania," Journal of the American Ceramic Society, vol. 73, no. 5, pp. 1172-1175, 1990.

[14] M. H. Teng, Y. C. Lai, and Y. T. Chen, "A computer program of master sintering curve model to accurately predict sintering results," Western Pacific Earth Sciences, vol. 2, no. 2, pp. 171-180, 2002.

[15] D. C. Blaine, S. Park, and R. M. German, "Master sintering curve for a two-phase material," in Proceedings of the 4th International
Conference on Science, Technology and Applications of Sintering, Grenoble, France, August 2005.

[16] D. Witkin, B. Q. Han, and E. J. Lavernia, "Mechanical behavior of ultrafine-grained cryomilled Al 5083 at elevated temperature," Journal of Materials Engineering and Performance, vol. 14, no. 4, pp. 519-527, 2005.

[17] Y. Sohn, "Diffusion in metals," in Smithells Metals Reference Book, W. F. Gale and T. C. Totemeier, Eds., chapter 13, Elsevier Butterworth-Heinemann, Oxford, UK, 8th edition, 2004.

[18] H. J. Frost and M. F. Ashby, Deformation-Mechanism Maps, vol. 1, Pergamon Press, New York, NY, USA, 1982. 

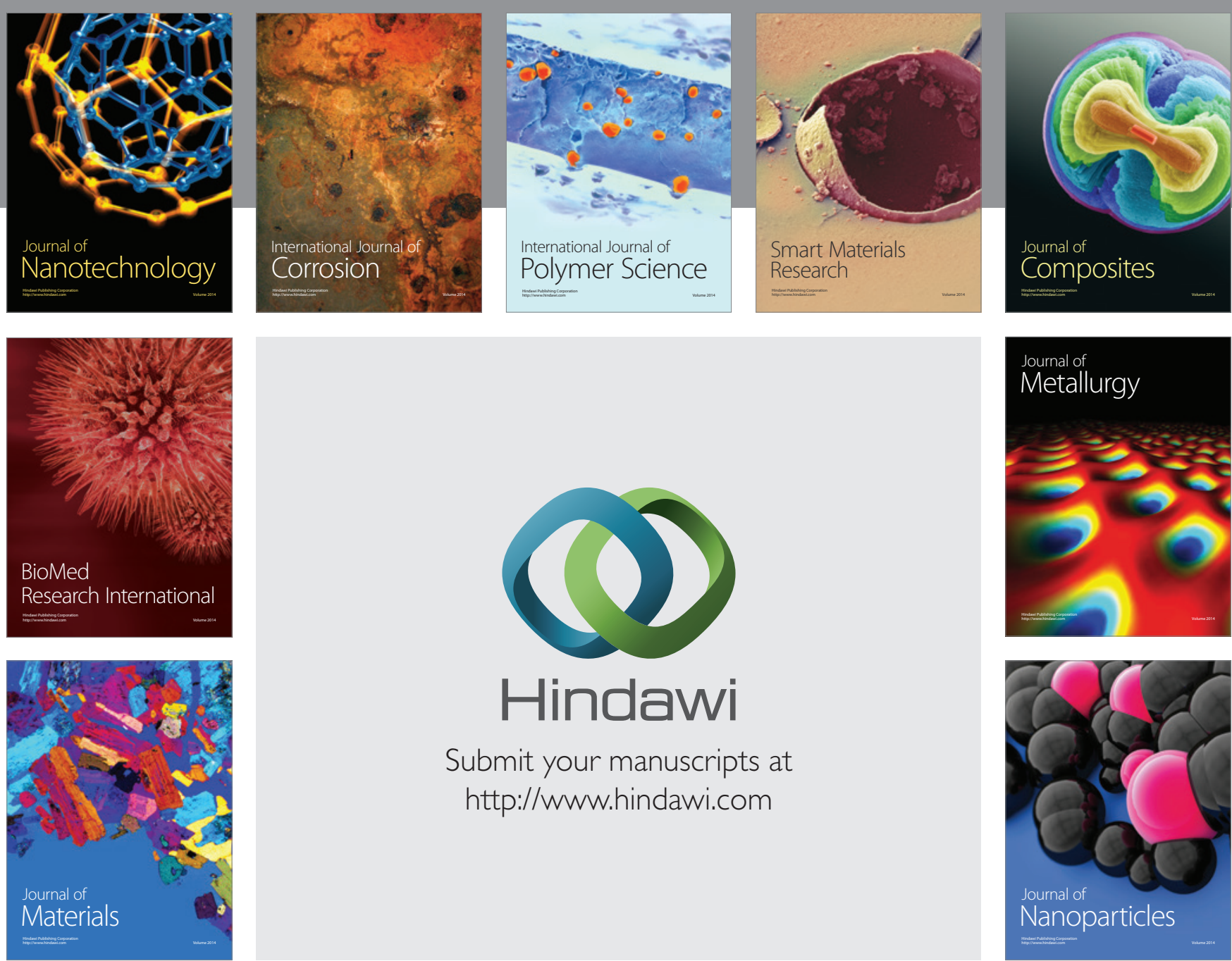

\section{Hindawi}

Submit your manuscripts at

http://www.hindawi.com

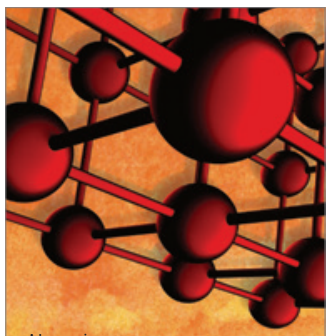

Materials Science and Engineering
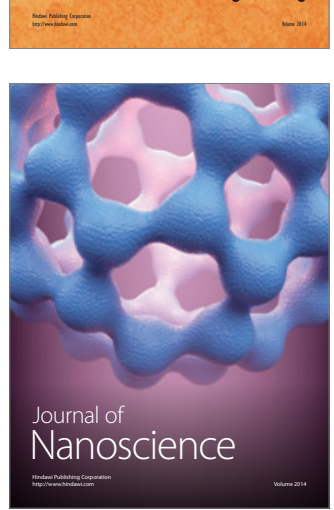
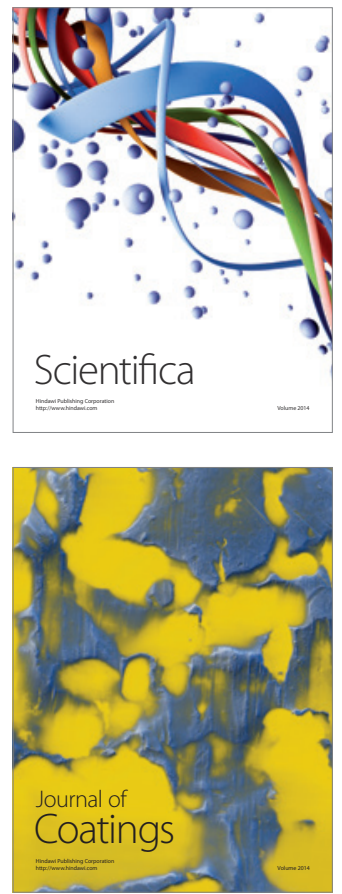
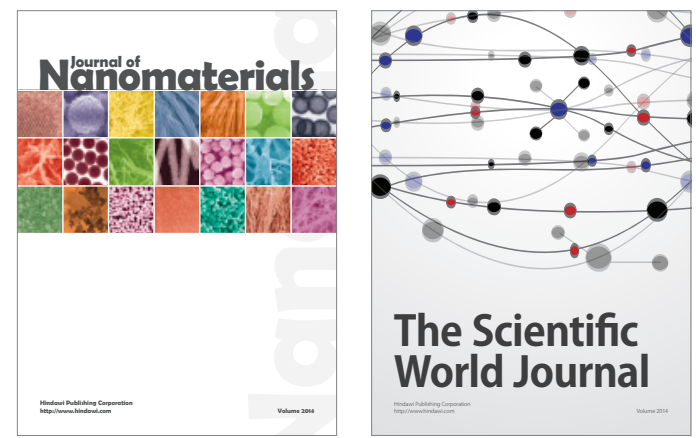

The Scientific World Journal
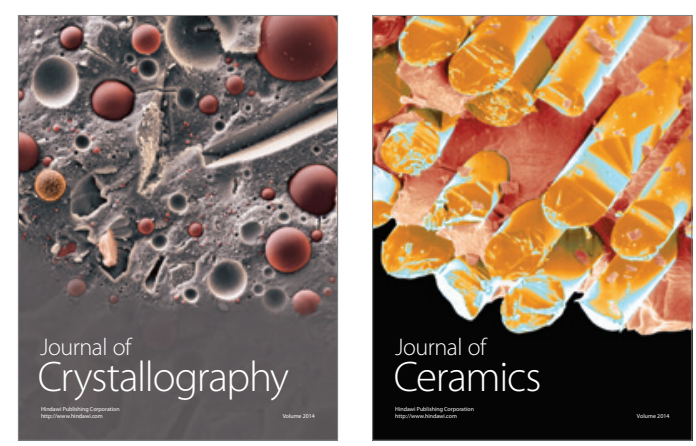
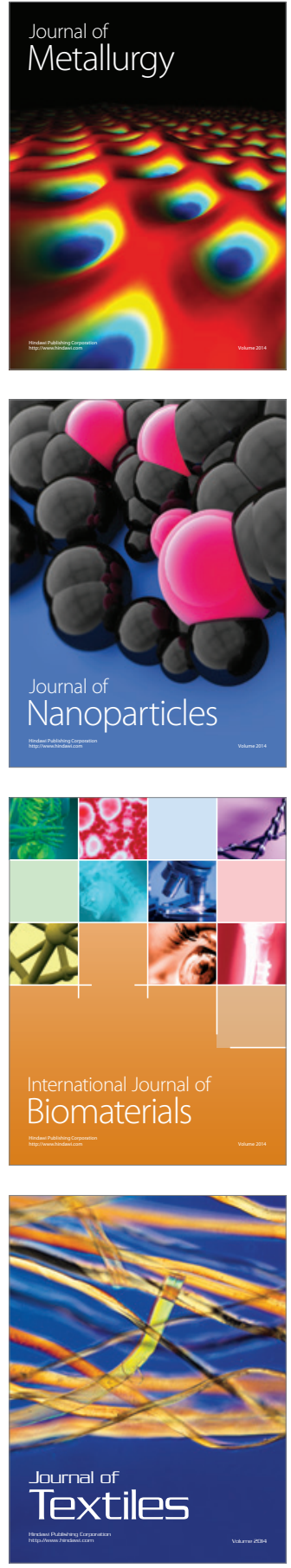